\title{
La correspondace de Jean Henri Samuel Formey (1711-1797): inventaire alphabétique, établi sous la direction de Jens Häseler
}

Paola Sosso

\section{(2) OpenEdition}

Journals

Edizione digitale

URL: http://journals.openedition.org/studifrancesi/36183

DOI: $10.4000 /$ studifrancesi.36183

ISSN: 2421-5856

\section{Editore}

Rosenberg \& Sellier

\section{Edizione cartacea}

Data di pubblicazione: 1 juillet 2005

Paginazione: 167-168

ISSN: 0039-2944

\section{Notizia bibliografica digitale}

Paola Sosso, «La correspondace de Jean Henri Samuel Formey (1717-1797): inventaire alphabétique, établi sous la direction de Jens Häseler», Studi Francesi [Online], 145 (XLIX | I) | 2005, online dal 30 novembre 2015, consultato il 18 avril 2021. URL: http://journals.openedition.org/studifrancesi/36183 ; DOI: https://doi.org/10.4000/studifrancesi.36183

Questo documento è stato generato automaticamente il 18 avril 2021.

\section{cc) (†) $\odot$}

Studi Francesi è distribuita con Licenza Creative Commons Attribuzione - Non commerciale - Non opere derivate 4.0 Internazionale. 


\title{
La correspondace de Jean Henri Samuel Formey (1711-1797): inventaire alphabétique, établi sous la direction de Jens Häseler
}

\author{
Paola Sosso
}

\section{NOTIZIA}

La correspondace de Jean Henri Samuel Formey (1711-1797): inventaire alphabétique, établi sous la direction de JENS HÄSELER, avec la Bibliographie des écrits de Jean Henri Samuel Formey établie par ROLF GEISSLER, Paris, Champion, 2003, («Vie des Huguenots»), pp. 475.

1 Il presente inventario riunisce per la prima volta tutto ciò che è disponibile della corrispondenza di Formey: si tratta innanzitutto delle quattro collezioni conservate alla Staatsbibliothek zu Berlin a Berlino e a Cracovia (fondo Formey, collezione Varnhagen, collezione Autographa e collezione Darmstaedter) provenienti in gran parte dalla successione di Formey; vengono inoltre fornite informazioni su numerose lettere di altre biblioteche o archivi (fra i più importanti, l'Académie des Sciences di San Pietroburgo e le collezioni olandesi, svizzere, francesi). La raccolta minuziosa di testi, la ricostruzione del cammino seguito da molti scritti, ripercorso nella prima parte dell'introduzione, ha consentito al curatore di inventariare un totale di 17.100 lettere scritte da 2.427 corrispondenti in sei lingue diverse, di cui 1200 scritte da Formey. La maggioranza delle lettere sono in francese, alcune in tedesco e in latino, altre in italiano, olandese e inglese. Molti scritti furono pubblicati quando Formey era ancora in vita: tra gli altri, quelli inviati a Francesco Algarotti e a Jacques Matter. Altri sono già stati raccolti nelle edizioni dell'epistolario di alcuni grandi autori del XVIII secolo, ad esempio nella correspondance di Voltaire curata da Théodore Besterman o in quella di Rousseau edita da Ralph A. Leigh. La seconda sezione dell'introduzione ricostruisce le 
tappe fondamentali della vita di Formey fin dai primi anni della sua carriera sotto Federico Guglielmo I e poi sotto Federico II. Dopo gli studi di teologia, iniziò la sua attività di pastore, accompagnata da un'immensa attività di scrittore di sermoni. Ebbe inizio nel 1731 la redazione di testi epistolari, destinati ad aumentare progressivamente con l'ampliarsi degli interessi dell'autore, diviso tra engagement pastorale e curiosità scientifiche e filosofiche. Formey mantenne viva in questi anni un'intensa collaborazione con molte riviste, e dal 1743 divenne membro della Società letteraria nell'ambito della quale fiorì il progetto di un dizionario filosofico.

2 Federico II, che desiderava dar lustro alla Società, fusa nel 1744 in un unico ente con l'Académie de Berlin, incarico Formey di redigere gli elogi di alcune personalità illustri, lo nominò dapprima storiografo ufficiale e poi, a partire dal 1748, segretario dell'Accademia. In questi anni iniziarono i rapporti epistolari con personaggi illustri di tutta Europa. Di particolare rilievo la corrispondenza con Maupertuis, che durò fino al 1759: essa fornisce elementi di grande interesse per ricostruire le vicende dell'Accademia relative a quegli anni. Da ricordare anche le numerose lettere a librai ed editori di varie nazioni, testimoni di un grande desiderio di divulgazione e di un radicato senso degli affari. Mancano purtroppo alcune annate dell'epistolario, ad esempio il 1752, anno dell'affaire del Docteur Akakia, periodo difficile in cui Formey rimase un po' in disparte rispetto agli eventi. Dal 1780 cominciò una lenta diminuzione dell'attività epistolare, che assunse toni diversi rispetto al passato: l'autore riprese e spiegò la sua attività di predicatore e illustrò liberamente le sue idee e i suoi progetti di riforma. Personaggio attivo, pastore, accademico, giornalista, insegnante e traduttore, Formey riflette nella sua vasta corrispondenza il ruolo centrale da lui svolto all'interno del secolo dei Lumi. In una delle ultime sezioni dell'Introduzione viene fornita la ripartizione geografica dei luoghi di spedizione delle lettere, la collocazione professionale dei corrispondenti, la classificazione e il raggruppamento delle lettere per corrispondenti e per periodo. Per le sue dimensioni, l'epistolario di Formey eguaglia quello di Albrecht von Haller e si avvicina a quello di Voltaire, anche se non dal punto di vista qualitativo: vi sono testi a carattere familiare, di tema religioso, filosofico, accademico, e una grande presenza di argomenti letterari. Tutto questo fa di Formey un "passeur d'informations": pensatore certo non originale, egli fu tuttavia grande divulgatore, in una posizione cardine tra il mondo delle lettere, il mondo accademico e il pubblico colto del suo secolo.

3 Carrefour delle nuove idee e dei ferventi dibattiti dell'epoca, la corrispondenza di Formey costituisce anche un passo fondamentale nella ricostruzione della dinamica che vide l'inserimento e l'evoluzione intellettuale di molti francesi, ugonotti o philosophes, nella Berlino del XVIII secolo: le lettere permettono infatti di definire, attraverso i vastissimi rapporti instaurati grazie alla scrittura, la trama di un panorama culturale ricco e variegato. Un dettagliato Avertissement chiarisce i principi seguiti nella presentazione dell'Inventaire e della Bibliographie. Lo stesso Formey aveva redatto più volte, tra il 1755 e il 1765, la bibliografia dei suoi testi: la maggior parte delle sue opere furono pubblicate in Germania, e questo spiega le lacune di alcuni grandi manuali bibliografici francesi come il Cioranescu e il Quérard. Anche le bibliografie moderne tedesche sono tuttavia incomplete, con la sola eccezione della parte riguardante gli scritti di carattere accademico. La gamma delle pubblicazioni incluse nella bibliografia include quasi tutti i generi letterari dell'epoca, dialoghi, lettere, sermoni, testi di carattere storico, trattati, saggi, dizionari e romanzi, a cui vanno aggiunte le numerose 
traduzioni. La bibliografia è divisa in varie rubriche all'interno delle quali la presentazione segue l'ordine cronologico: opere pubblicate separatamente dalla stesso Formey, opere da lui tradotte, scritti accademici, articoli pubblicati in volumi o su periodici, e, in ultimo, la lista dei testi falsamente attribuiti all'autore e alcuni manoscritti, fino alle edizioni moderne della corrispondenza. 